\title{
Verbesserung der Entmischung hyperspektraler Bilder durch den Einbezug räumlicher Information
}

Sebastian Bauer, Johannes Stefan, Matthias Michelsburg und Fernando Puente León Institut für Industrielle Informationstechnik, Karlsruher Institut für Technologie Tel.: 0721/608 44515, e-mail: sebastian.bauer@kit.edu

\section{Kurzfassung}

Im Gegensatz zu Grauwert- oder RGB-Bildern enthalten hyperspektrale Bilder statt ein bzw. drei Farbkanälen bis zu mehrere hundert Farbkanäle. Damit existiert bei allen betrachteten Wellenlängen eine vollständige Abbildung einer Szene. Aus diesem Grund ist hyperspektrale Bildverarbeitung auch als bildgebende Spektroskopie bekannt; interpretiert man das hyperspektrale Bild auf eine andere Art, liegt pro Pixel ein vollständiges Spektrum vor. Da viele Materialien eine charakteristische spektrale Signatur besitzen, ermöglicht die Ausnutzung der spektralen Information auch die Bestimmung der in einer Szene enthaltenen Materialien und ihre relativen Anteile (sogenanntes spektrales Entmischen oder auch Spectral Unmixing). Durch die Nutzung dieses optischen Messverfahrens lassen sich beispielsweise industrielle Sortierprozesse verbessern oder werden gar erst ermöglicht.

Es werden unüberwachtes und überwachtes Entmischen unterschieden: Beim überwachten Entmischen sind die spektralen Signaturen der Reinstoffe bekannt, während diese beim unüberwachten Entmischen im Prozess mitbestimmt werden müssen. Im Allgemeinen erfolgt die Entmischung pixelweise. Erst in den letzten Jahren wurden vermehrt Algorithmen vorgestellt [1,2], die auch die räumliche Nachbarschaft der Pixel berücksichtigen und damit die Entmischungsresultate verbessern, da sich die Materialanteile von Pixel zu Pixel meist nur wenig unterscheiden. Dadurch lassen sich die durch Messfehler und -rauschen auftretenden Fehler verringern.

In dieser Arbeit werden unterschiedliche Algorithmen untersucht, die durch 
den Einbezug räumlicher Information den unüberwachten Entmischungsprozess verbessern. Zum einen wurde basierend auf dem in [3] vorgestellten Ansatz ein Algorithmus entwickelt, der nicht nur konstante, sondern auch lineare Übergänge in den Materialanteilen von einem Pixel zu seinen Nachbarn modelliert. Der in [3] beschriebene Algorithmus fußt auf der Minimierung der Total Variation (interpretierbar als Approximation der ersten Ableitung) [4], während die Neuentwicklung die zweite Ableitung der AnteilsPixelübergänge minimiert. Zum anderen wurde aufgrund des hohen Rechenaufwands bei der Berechnung der beiden vorgenannten Ansätze die räumliche Information nicht direkt in den Entmischungsprozess integriert, sondern implizit durch das Einfügen von Zwischenschritten in der Berechnung berücksichtigt. Dies geschieht durch die Anwendung einiger aus der klassischen Bildverarbeitung bekannten Bildglättungsverfahren. Dadurch wird eine signifikante Rechenzeitersparnis realisiert, allerdings hängt das Ergebnis auch wesentlich von der Parameterwahl ab. Sowohl der direkte als auch der implizite Ansatz verbessern durch den Einbezug räumlicher Information die Entmischungsergebnisse zum Teil deutlich. Dies wird anhand von real aufgenommenen Daten demonstriert. Die aufgezeichneten Bildspektren stammen von speziell angefertigten Mischungen weißer Pulver. Auch auf die problemangepasste Vorverarbeitung der aufgezeichneten Bildspektren wird eingegangen.

\section{Einleitung}

Aus hyperspektralen Bildern lassen sich Informationen über die in einer Szene enthaltenen Materialien gewinnen. In der industriellen Sortierung existiert eine Reihe von Problemstellungen, die sich mit herkömmlichen RGB-Kameras nicht zufriedenstellend lösen lassen [5]. Insbesondere bei natürlich gewachsenen Produkten wie Obst oder Gemüse lassen sich Schäden oder Fremdmaterial nur sehr schwer bis gar nicht feststellen. Auch bei Mineralen ist die Farbe kein eindeutiges Sortierkriterium. Aus diesem Grund werden vermehrt hyperspektrale Sortieranlagen entwickelt, noch befinden sich aber erst wenige im tatsächlichen Industrieeinsatz. Diese Sortieranlagen bieten den Vorteil, dass sie schnell, berührungslos und zerstörungsfrei arbeiten. 


\section{Mathematische Grundlagen}

Zur Extraktion der Reinmaterialspektren aus den mit einer Hyperspektralkamera gewonnenen Mischspektren lässt sich das lineare Mischmodell verwenden. Es lautet

$$
\mathbf{Y}=\mathbf{M A}+\mathbf{N} \text {. }
$$

Dabei wird angenommen, dass sich die Spektren der Reinstoffe ungestört überlagern. Für eine physikalische Erklärung und weitere Mischmodelle siehe [6]. Die Bildmatrix $\mathbf{Y}(L \times I)$ stellt in Gleichung (1) das gemessene Hyperspektralbild dar. Es enthält jedes der $I$ Pixelspektren der Länge $L$, wobei $L$ die Anzahl der spektralen Abtastwerte beschreibt. Bei der Zerlegung der Bildmatrix in die Anteilsmatrix $\mathbf{A}(R \times I)$ und die Reinspektrenmatrix $\mathbf{M}(L \times R)$ mit der Materialanzahl $R$ ergibt sich die Schwierigkeit, dass sich die Bildmatrix als Produkt unendlich vieler möglicher Matrizen $\mathrm{A}$ und $\mathrm{M}$ darstellen lässt.

\subsection{Simultane Regularisierung}

Eine einfache Matrixzerlegung kann mittels des Least-Squares-Gütemaßes

$$
J_{\text {data }}(\mathbf{M}, \mathbf{A})=\|\mathbf{M A}-\mathbf{Y}\|_{F}^{2}
$$

mit der Frobenius-Norm $\|\cdot\|_{F}$ berechnet werden. Als zusätzliche Nebenbedingung lässt sich eine räumliche Regularisierung einführen [3]:

$$
J_{\mathrm{TV}, \text { sim }}=\|\mathbf{M A}-\mathbf{Y}\|_{F}^{2}+\lambda_{\mathrm{TV}, \operatorname{sim}} \sum_{i=1}^{I} \sum_{j \in \mathcal{N}(i)}\left\|\mathbf{a}_{i}-\mathbf{a}_{j}\right\|_{1} .
$$

Der Index TV,sim macht hierbei deutlich, dass die Regularisierung direkt (simultan) in den Entmischungsprozess einbezogen wird; TV steht für Total Variation, ein Gütemaß zum Entrauschen von Grauwertbildern. Dabei wird die räumliche Ableitung der Anteile minimiert und im Idealfall zu 0 gesetzt, wodurch sich ein konstanter Anteilsverlauf ergibt, während Kanten zwischen Anteilsregionen sehr gut erhalten bleiben. Der Parameter $\lambda_{\mathrm{TV} \text {,sim }}$ balanciert die Bedingung, dass die Matrizen A und $\mathbf{M}$ die gemessenen Daten möglichst gut erklären sollen und die Bedingung, dass sich die Anteile benachbarter Pixel (beschrieben durch die Menge $\mathcal{N}$ ) möglichst wenig unterscheiden sollen, aus. Für die Minimierung von 
(3) siehe [3]. Dieses Gütemaß wird von uns auf die Minimierung der zweiten Ableitung erweitert, wodurch auch lineare Übergänge zwischen den Anteilen benachbarter Pixel erlaubt werden:

$$
J_{\mathrm{HS}, \operatorname{sim}}=J_{\mathrm{data}}(\mathbf{M}, \mathbf{A})+\lambda_{\mathrm{HS}, \operatorname{sim}} R_{\mathrm{HS}, \operatorname{sim}}(\mathbf{A})
$$

mit der Regularisierung

$$
R_{\mathrm{HS}, \operatorname{sim}}(\mathbf{A})=\sum_{r=1}^{R} \sum_{n_{x}=1}^{N_{x}} \sum_{n_{y}=1}^{N_{y}}\left\|\mathbf{H}\left(\mathbf{A}_{r}\right)\left(n_{x}, n_{y}\right)\right\|_{\mathrm{F}} .
$$

HS steht hierbei für „Hesse“, da die Hesse-Matrix $\mathbf{H}$ die zweite Ableitung einer zweidimensionalen Funktion darstellt. Durch die Regularisierung nach Gleichung (5) wird berücksichtigt, dass die Summe der Frobenius-Normen aller Hesse-Matrizen aller Reinstoffe über alle Bildpixel möglichst gering bleibt. Das Argument $\mathbf{H}\left(\mathbf{A}_{r}\right)$ verdeutlicht, dass die Anteilsmatrix $\mathbf{A}(R \times I)$ aller Materialien zunächst in die Anteilsmatrizen $\mathbf{A}_{r}\left(N_{x} \times N_{y}\right)$ der einzelnen Materialien umgeformt werden muss. $\left(n_{x}, n_{y}\right)$ stellt die Pixelkoordinate im Bild dar, während $N_{x}$ und $N_{y}\left(I=N_{x} \cdot N_{y}\right)$ die Anzahl der Pixel in $x$ - bzw. $y$-Richtung beschreiben. Da das Gütemaß (4) und (5) differenzierbar ist, wird ein Gradientenverfahren zur Minimierung vorgeschlagen. Dieses hat eine ähnliche Struktur wie der Algorithmus zur Minimierung von (3) in [3]. Aus Platzgründen kann auf den exakten Algorithmus nicht näher eingegangen werden.

\subsection{Sequentielle Regularisierung}

Da die Algorithmen zur Minimierung der Gütemaße (3) und (4)/(5) relativ zeitaufwändig sind, wurde untersucht, ob sich die räumlichen Nachbarschaftsbedingungen zeiteffizienter einbeziehen lassen. Zur Minimierung des Optimierungsproblems (2) ohne räumliche Regularisierung wird oftmals, besonders in der Chemometrie, der Alternating Least-Squares (ALS)-Algorithmus verwendet. Dabei wird jeweils eine der Matrizen A und M konstant gehalten und iterativ nach der anderen minimiert. Ein Anwendungsbeispiel ist in [7] gegeben. Durch das Einfügen eines Bildglättungsschritts nach der Bestimmung der Anteilsmatrix A, der jeweils die Regularisierung nach den Gleichungen (3) und (5) 
auf die Anteilsmatrizen aller Stoffe anwendet, lassen sich die Nachbarschaftsinformationen ebenfalls einbeziehen. Diese Methode nennen wir sequentielles TV und HS. Die verwendeten Algorithmen finden sich in [8] und [9].

\subsection{Spektrenvorverarbeitung}

$\mathrm{Da}$ die sequentiellen Verfahren wesentlich anfälliger gegenüber Parameterschwankungen (Regularisierungsparameter, Iterationenzahl, Anfangswerte etc.) als die simultanen Verfahren sind und bei Verwendung der Rohspektren zu keiner plausiblen Lösung kommen, müssen die gewonnenen Bildspektren vorverarbeitet werden. Dabei wird jedes Spektrum durch ein individuelles Polynom siebten Grades approximiert und dieses Polynom von dem jeweiligen Spektrum abgezogen. Die so gebildete Differenz wird als neues „Spektrum“ in die Minimierungsalgorithmen einbezogen.

\section{Entmischungsergebnisse bei aufgezeichneten Daten}

\subsection{Datenaufzeichnung}

Zur Evaluation der oben beschriebenen Algorithmen wurden mit einer hyperspektralen Zeilenkamera (Specim SP-SWIR-LVDS-100-N25E SWIR) Aufnahmen weißer Pulvermischungen aufgezeichnet. Dabei sind die Spektren Reflektanzspektren, geben also an, bei welcher Wellenlänge welcher Anteil des eingestrahlten Lichts reflektiert wird. Für die Pulvermischungen wurden die Stoffe Milchzucker, Speisestärke, Puderzucker, Magnesiumcarbonat und Modelliergips in Anteilen von Vielfachen von einem Achtel vermischt.

\subsection{Ergebnisse}

Die Anteilsmatrizen sowie die ermittelten Reinspektren bei unüberwachter Entmischung sind in Bild $1 \mathrm{zu}$ sehen. Es sei darauf hingewiesen, dass der ALSAlgorithmus bereits akzeptable Ergebnisse liefert und die sequentiellen Entmischungsmethoden diese lediglich noch weiter verbessern. Der Fakt, dass der Bildverarbeitungsschritt das ALS-Ergebnis weiter verbessert, wurde durch Analyse des Fehlers zwischen wahren und geschätzten Anteilen verifiziert. Allerdings ist die Güte des ALS-Ergebnisses der geschickten Spektrenvorverarbei- 

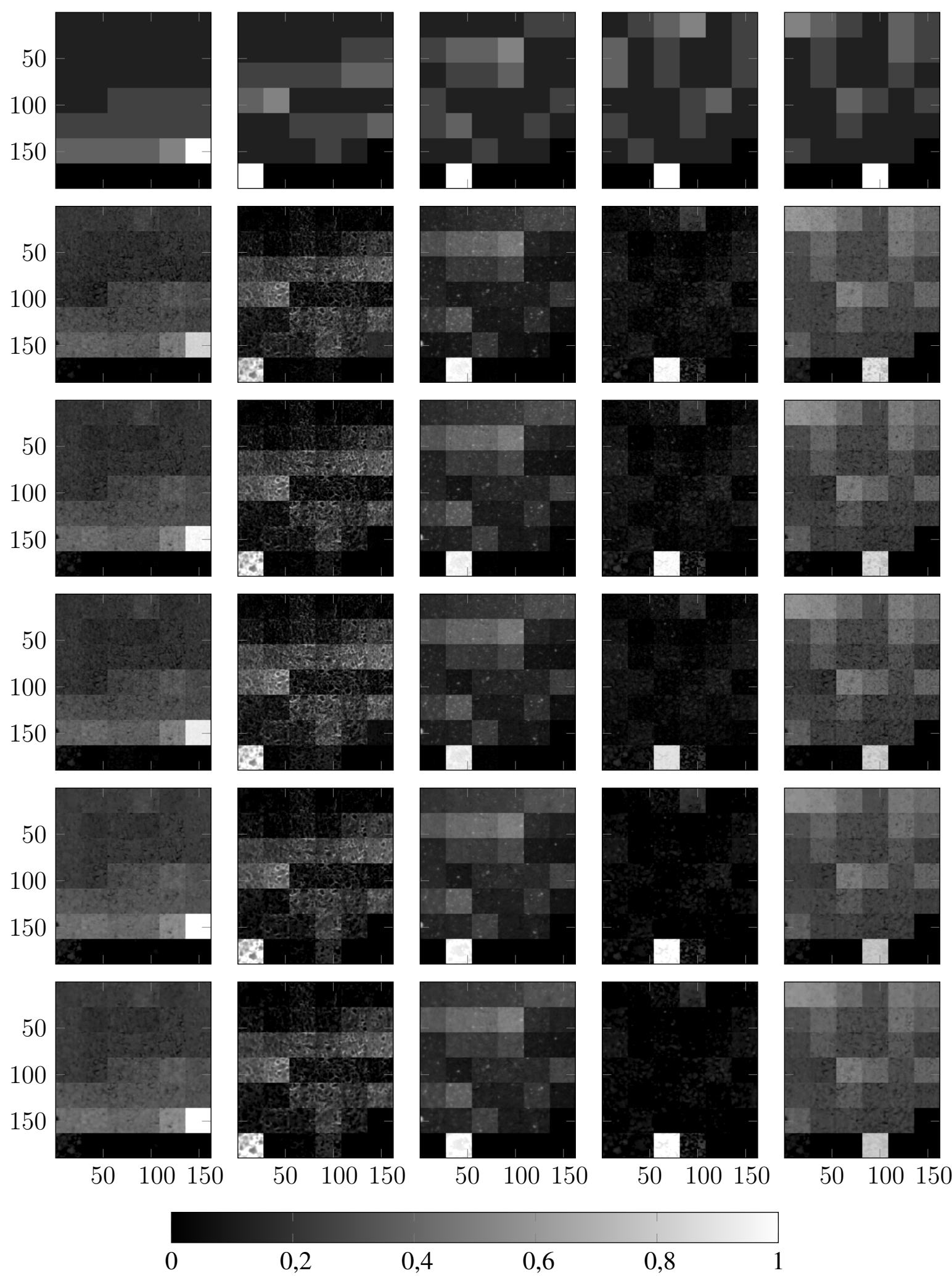

Bild 1: Anteilsmatrizen der Reinstoffe. Die Spalten gehören dabei jeweils zu einem Reinstoff. In der ersten Zeile sind die Ground-Truth-Anteile, in der zweiten die Ergebnisse der simultanen TV-Entmischung und in der dritten die Ergebnisse der simultanen HS-Entmischung zu sehen. Die vierte Zeile stellt die Entmischungsergebnisse von ALS dar, während die fünfte und sechste Zeile die sequentiellen TV- bzw. HS-Ergebnisse zeigen. Die Zahlen an den $x$ - und $y$-Achsen stellen die Pixelindizes dar. 
tung nach Abschnitt $2.3 \mathrm{zu}$ verdanken. Wird mit einer anderen oder ohne Vorverarbeitung gearbeitet, so fällt die Verbesserung durch die Regularisierungsmethoden deutlich größer aus bzw. der ALS-Algorithmus konvergiert gar zu einer falschen Lösung. Bild 2 zeigt die Spektren der Reinstoffe, die im Laufe des Entmischungsprozesses parallel zu den Anteilsmatrizen in Bild 1 ermittelt wurden. Sowohl die simultanen als auch die sequentiellen Versionen der TVund der HS-Methode sind sehr anfällig gegenüber der Wahl des Regularisierungsparameters $\lambda$, wobei die simultanen Versionen etwas weniger empfindlich sind. Dafür sind die sequentiellen Verfahren ca. 20-25\% schneller als die simultanen Verfahren. Die Auswahl des Parameters, die zu Entmischungsergebnissen akzeptabler Qualität führt, ist unter anderem abhängig von der jeweiligen Problemstellung (Bildgröße, vorhandene Reinspektren etc.).

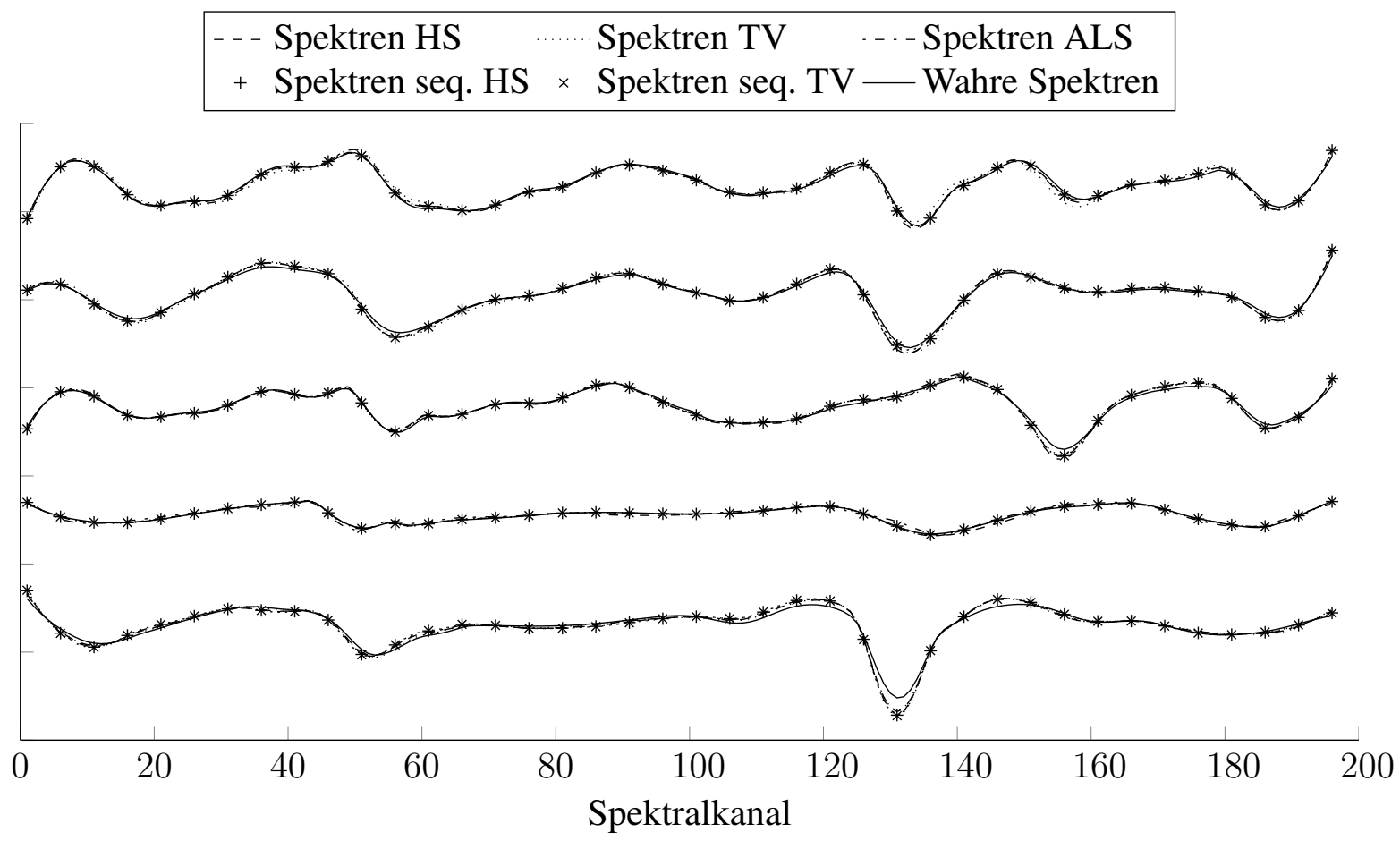

Bild 2: Ermittelte Reinspektren. Die Spektren wurden in $y$-Richtung abgesetzt, um die Verläufe zu verdeutlichen. Das oberste Spektrum gehört zum Stoff der ersten Spalte der Anteilsmatrizen in Bild 1, das zweitoberste zur zweiten Spalte usw.

\section{Zusammenfassung und Ausblick}

In dieser Arbeit wurde untersucht, inwiefern der Einbezug räumlicher Information die Entmischungsergebnisse hyperspektraler Bilder verbessern kann. Es 
wurden mehrere Verfahren, die die räumliche Information auf unterschiedliche Weise berücksichtigen, entwickelt bzw. vorgestellt. Der Einbezug der räumlichen Information verbessert die Qualität der Entmischungsergebnisse zum Teil deutlich, allerdings hat die Wahl des Regularisierungsparameters großen Einfluss auf diese Qualität. Es wurde untersucht, inwiefern sich die Berechnungsdauer bei den regularisierenden Verfahren verkürzen lässt. Zukünftige Arbeiten haben den Einbezug von Unmixing-Methoden in die hyperspektrale Bildverarbeitung für die industrielle Sortierung zum Ziel.

\section{Literatur}

[1] K. Canham, A. Schlamm, A. Ziemann, B. Basener und D. Messinger, "Spatially adaptive hyperspectral unmixing", Geoscience and Remote Sensing, IEEE Transactions on 49(11), S. 4248-4262, 2011.

[2] O. Eches, N. Dobigeon und J.-Y. Tourneret, "Enhancing hyperspectral image unmixing with spatial correlations", Geoscience and Remote Sensing, IEEE Transactions on 49(11), S. 4239-4247, 2011.

[3] A. Zymnis, S. Kim, J. Skaf, M. Parente und S. Boyd, "Hyperspectral image unmixing via alternating projected subgradients", in Signals, Systems and Computers, 2007. ACSSC 2007. Conference Record of the Forty-First Asilomar Conference on, S. 1164-1168, 2007.

[4] L. I. Rudin, S. Osher und E. Fatemi, "Nonlinear total variation based noise removal algorithms", Physica D: Nonlinear Phenomena 60(14), S. 259-268, 1992.

[5] M. Michelsburg, R. Gruna, K.-U. Vieth und F. Puente León, "Spektrale Bandselektion beim Entwurf automatischer Sortieranlagen", in Forum Bildverarbeitung 2010, S. 389-400, KIT Scientific Publishing, 2010.

[6] N. Keshava und J. Mustard, "Spectral unmixing", Signal Processing Magazine, IEEE 19(1), S. 44-57, 2002.

[7] A. d. Juan, R. Tauler, R. Dyson, C. Marcolli, M. Rault und M. Maeder, "Spectroscopic imaging and chemometrics: a powerful combination for global and local sample analysis", TrAC Trends in Analytical Chemistry 23(1), S. 70-79, 2004.

[8] http://www.mathworks.com/matlabcentral/fileexchange/16204-toolboxsparse optmization/content/toolbox_optim/tests/html/test_tv_lagrangian.html.

[9] http://bigwww.epfl.ch/stamatis/publications.html. 